P. Stefanini made a series of three hundred slides; these are of special educational value and they have been used during numerous refresher courses on the subject "sky observation."

The Society is also represented in certain commissions of the Ministry of Public Education for the compilation of new programs for physics and natural science.

\title{
ASTRONOMY ACTIVITIES OF THE AMERICAN ASSOCIATION OF PHYSICS TEACHERS
}

Robert J. Dukes, Jr.

Physics Department, The College of Charleston, Charleston, South Carolina 29424, U.S.A.

The American Association of Physics Teachers (AAPT) was founded in 1930 by members of the American Physical Society whose main interest was the teaching of physics. Since then its primary goal has remained the improvement of physics teaching at all levels. The Association publishes two journals (American Journal of Physics and The Physics Teacher) and a bulletin (The Announcer). The American Journal of Physics is intended for papers concerning physics teaching at the level of colleges and universities in the United States while The Physics Teacher is concerned primarily with introductory physics, including high-school courses. Two national meetings are held annually as well as numerous regional meetings.

Fortunately for astronomy education, AAPT's definition of physics has from the first included astrophysics. Early papers in the American Journal of Physics (then called the American Physics Teacher) included "Epsilon Aurigae, Colossus Among Stars, a Story of Co-operative Research in Photometry, Spectroscopy, and the Theory of Gases" by Struve and Lemon (1938) and "Mnemonic for Bethe's Solar Energy Reactions" by Randall (1948).

In 1983 the AAPT Executive Board established a Committee on Astronomy Education whose primary duty was to provide information for physicists who are teaching astronomy. This committee has sponsored a number of symposia at various meetings of the Association as well as working to publicize activities of other societies concerned with astronomy education among AAPT members. A survey of AAPT national meetings from 1971 through 1983 (Dukes and Strauch, 1984) showed that approximately five per cent of all papers presented at national AAPT meetings concerned astronomy (approximately 170 papers on astronomy out of a total of 3000 papers). This ratio has remained approximately constant to the present when 20 of the approximately 400 papers presented at the two 1988 national meetings concerned astronomy. Also in 1988 five papers on astronomy were published in The Physics Teacher and approximately 18 papers in the American Journal of Physics. 
Finally the American Journal of Physics publishes a series of annotated bibliographies called "Resource Letters." Two have been directly concerned with astronomy education. The first of these was an extensive listing of astronomy education resources (Berendzen and DeVorkin, 1973). The second listed laboratory exercises for astronomy (Kruglak, 1976). Others that are of interest to astronomers include those on cosmology (Ryan and Shepley, 1976), black holes (Detweiler, 1981), x-ray astronomy (Canizares, 1984), and cosmology and particle physics (Lindley, Kolb, and Schramm, 1988).

In summary, the American Association of Physics Teachers through its various activities is continually working towards improving astronomy education in this country. Its journals are receptive to manuscripts on topics similar to many of those presented at this meeting. I encourage you to submit papers on astronomy education to them.

\section{References}

Berendzen, R., and DeVorkin, D. 1973, "Resource Letter EMAA-1: Educational Materials in Astronomy and Astrophysics," Am. J. Phys., 41, 783-808.

Canizares, C.R. 1984, "Resource Letter XRA-1: X-Ray Astronomy," Am. J. Phys., 52, 111-19.

Detweiler, S. 1981, "Resource Letter BH-1: Black Holes," Am. J. Phys, , 49, 915-25.

Dukes, R.J., Jr., and Strauch, K. 1984, "Trends in Physics Teaching," Physics Teacher, 22, 446-448.

Kruglak, H. 1976, "Laboratory Experiences for Elementary Astronomy," Am. J. Phys., 44, 828-33.

Lindley, D., Kolb, E.W., and Schramm, D.N. 1988, "Resource Letter CCP-1: Cosmology and Particle Physics," Am. J. Phys., 56, 492-501.

Randall, C.A. 1948, "Mnemonic for Bethe's Solar-Energy Reaction," Am. J. Phys., $16,56$.

Ryan, M.P., Jr., and Shepley, L.C. 1976, "Resource Letter RC-1: Cosmology," Am. J. Phys., 44, 223-30.

Struve, O. and Lemon, H.B. 1938, "Epsilon Aurigae, Colossus Among Stars, a Story of Co-operative Research in Photometry, Spectroscopy, and the Theory of Gases," Am. J. Phys., 6, 123.

\section{Comment}

Jay M. Pasachoff: We have proposed in the AAPT Astronomy Education Committee, that the organization's name be changed to "The Association of Astronomy and Physics Teachers." This choice would retain the current initials and logo. Though the request did not pass at the highest levels, it is being repeated and may well be granted eventually. 


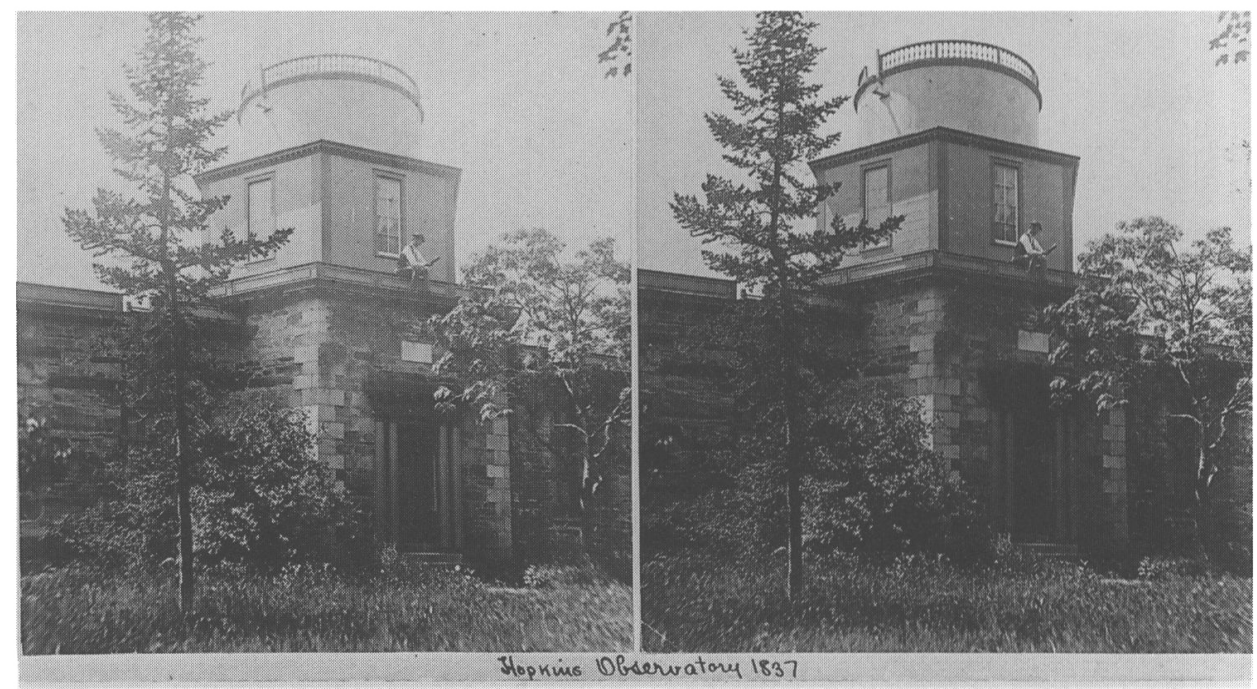

Stereoscopic view of the Hopkins Observatory, photographer unknown. Williamsiana Collection, Williams College. Further information on page 432. 